\title{
Left temporal lobe epilepsy patients without Hippocampal Sclerosis: A DTI and VBM study
}

Panagiotis Tsialios $^{1 *}$, Efstratios Karavasilis ${ }^{1}$, Irene Karanasiou ${ }^{2}$, Anastasios Bonakis ${ }^{3}$, Georgios Velonakis ${ }^{1}$, Efstathios Efstathopoulos ${ }^{1}$ Nikolaos Kelekis ${ }^{1}$ and Matilda-Anna Papathanasiou ${ }^{1}$

${ }^{1}$ Second Department of Radiology, Attikon University Hospital, Medical School, National and Kapodistrian University, 1 Rimini Str., Haidari, 12462 Athens, Greece

${ }^{2}$ Hellenic Military Academy, Varis - Koropiou Avenue, Vari, 16673 Athens, Greece

${ }^{3}$ Second Department of Neurology, Attikon University Hospital, Medical School, National and Kapodistrian University, 1 Rimini Str., Haidari, 12462 Athens, Greece

\begin{abstract}
Approximately, 30\% of temporal-lobe-epilepsy patients have no identified epiloptegenic finding on conventional brain MRI. The underlying pathological mechanism seems to be different when the laterality of seizure onset is considered. Thus, we examined the gray and white matter structural integrity of non-lesional left temporal lobe epilepsy patients. 16 left non-lesional temporal lobe epilepsy patients and 18 healthy controls underwent brain MRI protocol, including the conventional brain imaging epilepsy protocol along with 3D-T1 high resolution and diffusion tensor imaging sequences. The structural integrity was investigated using between-groups whole-brain voxel-based morphometry and reconstructing $20 \mathrm{WM}$ fiber bundles using diffusion tensor imaging tractography. Compared to healthy controls, patients presented gray matter atrophies in temporal, limbic and widespread extra-temporal regions, including occipital, parietal and frontal lobes as well as structures of the cerebellum. Moreover, fractional anisotropy decreases were detected in arcuate, cingulum, fornix, inferior longitudinal fasciculus, inferior fronto-occipital fasciculus and uncinate fasciculus bundles. All affected tracts were ipsilateral to the seizure focus. Reduced axial diffusivity values were also observed for both left arcuate and left cingulate hippocampal part tracts in the patient group. Concluding, the present study revealed temporal, limbic and widespread extra-temporal gray matter atrophies as well as ipsilateral, widespread alterations of temporal and extra-temporal white matter fiber tracts, in non-lesional left temporal lobe epilepsy patients compared to healthy controls, shedding light on a complex network which might be associated with left temporal lobe epilepsy discharges.
\end{abstract}

\section{Introduction}

Temporal lobe epilepsy (TLE) constitutes the most common form of focal epilepsy. Voxel-based morphometry (VBM) and diffusion tensor imaging (DTI) neuroimaging techniques can shed light on the underlying pathological mechanism. The worldwide ENIGMA mega-analysis study using advanced post-processing neuroimaging techniques has clearly identified the gray matter (GM) structural abnormalities in TLE patients with hippocampal sclerosis (HS). Although, non-lesional TLE (TLE-NL) patients were included in a generalized "all-other-epilepsies" cohort, researchers suggested future sufficiently powered studies using strict inclusion criteria to delineate this subtype syndrome fingerprint [1]. TLE-NL patients with negative conventional brain magnetic resonance imaging (MRI) consist approximately the $30 \%$ of patients who manifest TLE [2-4]. The absence of lesions on conventional MRI provokes inherent difficulties in detecting the epileptogenic onset zone, a very challenging case, particularly when the patient is a candidate for surgical management [5,6]. Moreover, TLE-NL patients are characterized by different clinical features compared to patients with HS (TLE-HS) $[2,4,7,8]$. This might reveal structural network differentiations between TLE-NL and TLEHS patients $[9,10]$.

In addition, the affected TLE-NL patients' structural network seems to be different when the laterality is considered [1,5,11-13]. Left TLE patients (LTLE) have shown major cognitive deficits $[14,15]$ that can be linked with the more widespread GM and white matter (WM) structural changes compared to right TLE patients (RTLE) [1,5,11,16,17]. Developmental factors and hemisphere vascular differences can be accounted for the vulnerability of the left hemisphere and partially explain these reported changes between RTLE and LTLE patients [1820]. Therefore, the underlying mechanism that differentiates these two apparently same neurological diseases is under investigation and slightly studied. To the best of our knowledge, concerning GM, there is a unique study in which temporal and extra-temporal GM changes were detected in LTLE-NL patients [5]. Additionally, concerning WM, the only available published study, revealed ipsilateral microstructural WM abnormalities in LTLE-NL patients [13]. Thus, in this study we try to shed light on the affected structural network of LTLE-NL patients, investigating both GM and WM integrity using advanced neuroimaging techniques in the same patient group with a strictly lateralized epileptic focus, left temporal, in the conventional electroencephalogram (EEG).

*Correspondence to: Panagiotis Tsialios, Second Department of Radiology, Attikon University Hospital, Medical School, National and Kapodistrian University, 1 Rimini Str., Haidari, 12462 Athens, Greece, Tel: +30 2114116976; Fax: +30 2130290587; E-mail: ptsialios@gmail.com

Key words: diffusion tensor imaging, magnetic resonance imaging (MRI), MRI negative epilepsy, temporal lobe epilepsy, voxel-based morphometry

Received: July 27, 2020; Accepted: August 11, 2020; Published: August 16, 2020 


\section{Materials and methods}

\section{Subjects}

In this study, 16 right-handed LTLE-NL patients were recruited (mean age: $30.69 \pm 11.40$ years, 8 females). Two RTLE-NL patients were excluded from this study in order to render the sample homogenous. The recruitment was conducted in the Attikon University Hospital (Department of Epileptology). The diagnosis was made by an expert on epilepsy, based on the presence of focal seizures consistent with TLE and focal epileptiform discharge in temporal areas on a conventional EEG. The presence of extra-temporal or multifocal epileptic foci, central nervous system disease other than epilepsy, history of serious head trauma, alcohol and drug abuse and contraindications to MRI examinations were considered exclusion criteria. Moreover, 18 healthy, right-handed, age and gender matched volunteers of the same ethnicity (Greek) as the patients and with no previous history of neurological or psychiatric disorders were recruited as a control group (mean age: 28.67 \pm 7.50 years, 11 females). Table 1 presents demographic and clinical parameters. The study was approved by the Bioethics Committee of the Attikon University Hospital and was conducted in accordance with the Helsinki Declaration of 1975.

\section{MRI acquisition}

All participants underwent a whole-brain high resolution 3D-T1weighted (HR_3DT1w, acquisition matrix: $1 \mathrm{~mm} \times 1 \mathrm{~mm} \times 1 \mathrm{~mm}$, repetition time: $9.9 \mathrm{~ms}$, echo-time: $3.7 \mathrm{~ms}$, flip angle: $7^{0}$ and sagittal orientation), and 32-directional DTI (acquisition matrix: $2 \mathrm{~mm} \times 2$ $\mathrm{mm} \times 2 \mathrm{~mm}$, repetition time: $7743 \mathrm{~ms}$, echo-time: $70 \mathrm{~ms}, 32$ diffusion encoding directions, two b values: $0 \mathrm{~s} / \mathrm{mm}^{2}$ and $1000 \mathrm{~s} / \mathrm{mm}^{2}$ and axial orientation) protocol on a 3T Philips Achieva-Tx MR scanner (Philips, Best, The Netherlands) equipped with an eight-channel head coil.
High resolution T2-weighted, T2-weighted fluid-attenuated inversion recovery and T1-weighted inversion recovery sequences were also acquired to identify lesions in patient group. The interpretation of the acquired images was conducted by two experienced neuroradiologists. The acquired sequences and their parameters are summarized in Table 2.

\section{Whole-brain VBM analysis}

Preprocessing and statistical analysis were carried out using the Statistical Parametric Mapping (SPM12; Wellcome Department of Cognitive Neurology, www.fil.ion.ucl.ac.uk/spm/software/spm12) and its extension toolbox in MATLAB R2018b, Computational Anatomy Toolbox (CAT12; The Structural Brain Mapping Group, University of Jena, http://www.neuro.uni-jena.de/cat/). In brief, preprocessing is summarized in the following steps: T1-weighted images were spatially normalized to the Montreal Neurological Institute space using the default International Consortium for Brain Mapping-European Brains template and segmented into GM, WM and cerebro-spinal fluid (CSF) tissues. The total intracranial volume (TIV) was estimated. GM images were smoothed with a $4 \mathrm{~mm}$ full-width-at-half-maximum (FWHM) isotropic Gaussian kernel in order to remove large signal discrepancies between neighboring voxels [21].

\section{DTI, Tractography analysis}

The acquired DTI data were processed and analyzed using ExploreDTI_v4.8.6 [22]. Initially, diffusion-weighted (DW) images were corrected for Gibbs ringing artifacts which are typically appearing between brain tissue boundaries [23]. Diffusion tensors were estimated using a robust fitting algorithm (REKINDLE) [24]. DTI datasets were corrected for subject motion, eddy current induced distortions and susceptibility artifacts due to field inhomogeneities related to the echoplanar imaging (EPI) sequence [25]. The resultant undistorted images

Table 1. Demographics and clinical parameters of subjects

\begin{tabular}{|c|c|c|c|}
\hline & Patients & Controls & \multicolumn{1}{c|}{ Statistics } \\
\hline No. & 16 & $7 / 11$ & $X^{2}=0.424 ; p=0.515^{*}$ \\
\hline Gender (male/female) & $8 / 8$ & $\begin{array}{c}28.67 \pm 7.50 \\
\text { (range: } 19-44)\end{array}$ & \\
\hline Age (years) & $30.69 \pm 11.40$ (range: $17-50)$ & \\
\hline Age at seizure onset (years) & $19.91 \pm 12.18$ (range: $1-47)$ & - \\
\hline Disease duration (years) & $\begin{array}{c}10.78 \pm 11.51 \\
\text { (range: } 1-33)\end{array}$ & - \\
\hline
\end{tabular}

Pearson chi-square test.

$\dagger$ Mann-whitney u test among groups.

Data are mean \pm standard deviation, except for the number of participants and gender distribution. The statistical significance level was set at $\mathrm{p}=0.05$

Table 2. Parameters of the acquired sequences

\begin{tabular}{|c|c|c|c|c|c|}
\hline \multirow{2}{*}{ Sequence Parameters } & \multicolumn{5}{|c|}{ Sequences } \\
\hline & 3D-T1-weighted & DTI-EPI* & T2-weighted & T2-weighted FLAIR & T1-weighted IR \\
\hline TR (ms) & 9.9 & 7736 & 3000 & 4800 & 3977 \\
\hline $\mathrm{TE}(\mathrm{ms})$ & 3.7 & 70 & 90 & 366 & 13 \\
\hline IR (ms) & - & - & - & 1650 & 400 \\
\hline Flip angle & $7^{\circ}$ & $90^{\circ}$ & $90^{\circ}$ & $90^{\circ}$ & $90^{\circ}$ \\
\hline Voxel-size $\left(\mathrm{mm}^{3}\right)$ & $1 \times 1 \times 1$ & $2 \times 2 \times 2$ & - & $1.4 \times 1.4 \times 1.4$ & - \\
\hline Slices & 170 & 75 & 44 & 254 & 65 \\
\hline Acquisition Matrix & $255 \times 240$ & $128 \times 126$ & $340 \times 255$ & $356 \times 358$ & $216 \times 214$ \\
\hline FOV $\left(\mathrm{cm}^{2}\right)$ & $25 \times 25$ & $25.6 \times 25.6$ & $22 \times 22$ & $25 \times 25$ & $22 \times 22$ \\
\hline Acquisition time (minutes) & $5: 59$ & $5: 37$ & $3: 48$ & $5: 22$ & $3: 51$ \\
\hline
\end{tabular}

DTI: Diffusion Tensor Imaging, EPI: Echo-Planar Imaging, FLAIR: Fluid-Attenuated Inversion Recovery, IR: Inversion Recovery, TR: Repetition Time, TE: Echo Time and FOV: Field-of-View. ${ }^{*}$ Two b-values, $0 \mathrm{~s} / \mathrm{mm} 2$ and $1000 \mathrm{~s} / \mathrm{mm} 2$ 
were visually inspected before the next step [26]. Subsequently, wholebrain tractography was conducted for each subject using a deterministic streamline approach with seed fractional anisotropy (FA) threshold of 0.2. Twenty major WM fiber tracts (forceps major-FMajor; forceps minor-FMinor; cingulum cingulate gyrus part-CCGP; cingulum hippocampal part-CHP; cortico-spinal tract-CST; fornix; anterior thalamic radiation-ATR; arcuate fasciculus-AF; inferior longitudinal fasciculus-ILF; inferior fronto-occipital fasciculus-IFOF and uncinate fasciculus-UNCF) were reconstructed bilaterally using regions of interest (ROIs) approach $[27,28]$. These ROIs were manually designed on the native color FA map of a representative healthy subject. Using the above-mentioned subject as a template, ROIs were automatically registrated and reconstructed for both patients and controls to resolve user-related variations due to the manual ROI definition. For each tract, FA, mean diffusivity (MD), axial diffusivity (AD), and radial diffusivity (RD) were extracted. Figure 1 presents a schematic overview of tractography analysis workflow.

\section{Statistical analysis}

Differences in demographics and clinical parameters were estimated using Pearson Chi-Square for gender and Mann-Whitney
$\mathrm{U}$ test for age. A two-sample t-test was conducted using SPM12 second level analysis with TIV, age and gender as nuisance variables, in order to assess statistically significant group differences in wholebrain gray matter volume (GMV). In view of the fact that cluster-size distribution depends on the local smoothness of data, a non-stationary cluster correction based on voxel-level $p<0.001$ (uncorrected) and a threshold of minimum expected voxels per cluster of the order of $\mathrm{k}=15$ voxels were implemented [29,30]. Concerning DTI metrics, multivariate analyses of variances (MANOVAs) were used in order to estimate differences in WM fiber tracts between LTLE-NL patients and healthy controls (HC) ipsilateral and contralateral to the seizure focus. Furthermore, differences in DTI metrics for the pair of Fmajor and Fminor tracts were evaluated with a separate MANOVA due to the fact that these two tracts extends to both brain hemispheres. A Wilks' Lambda $(\mathrm{p}<0.05)$ multivariate analysis, was followed by univariate analyses (ANOVAs) corrected for multiple comparisons (Bonferroni adjustments), to identify the WM fiber tracts that contribute to each statistically significant MANOVA. In our study the level of statistical significance of the ANOVAs was set at $\mathrm{p}<0.05$ because there was one dependent variable. All statistical analyses were performed using the Statistical Package for Social Sciences (SPSS v25, Chicago, U.S.A.).

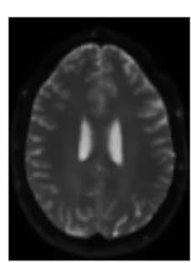

DW images
Gibbs ringing correction

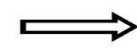

Data

reconstruction

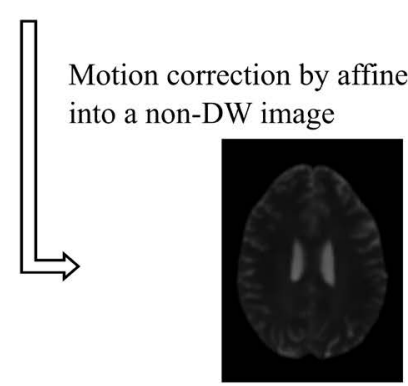

non-DW image

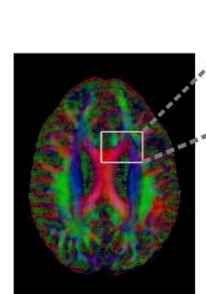

Diffusion tensors

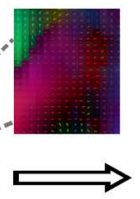

Eddy current $\&$ susceptibility artifacts distortions correction by registering into an undistorted image

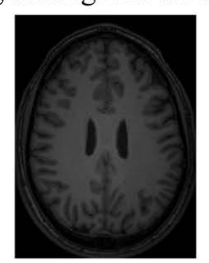

T1-weighted image

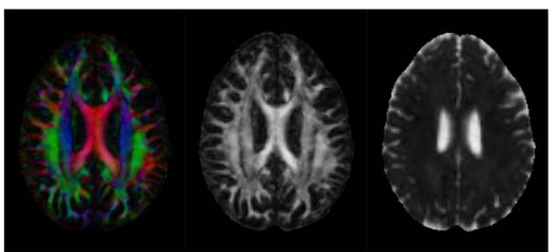

Color FA map $\quad$ FA map $\quad$ MD map

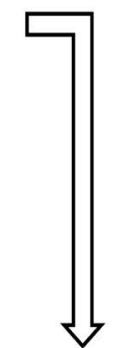

Visual inspection

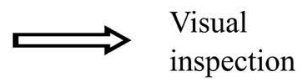

WM fiber tracts reconstruction

\begin{abstract}
Automated atlas or template tractography

DTI metrics extraction (FA, MD, AD and RD) for each tract
\end{abstract} e.g. L IFOF

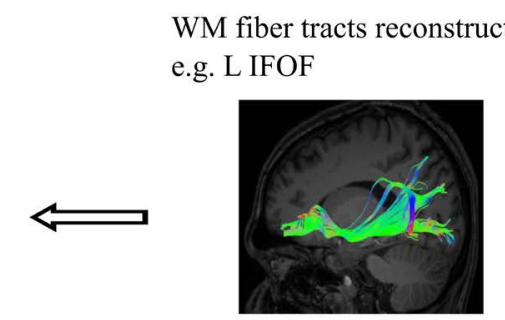

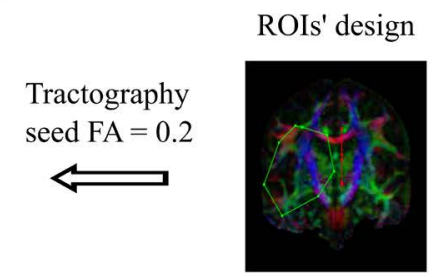

Figure 1. Schematic overview of Tractography analysis workflow. DW images are corrected for Gibbs ringing artifacts, motion artifacts, eddy current distortions and susceptibility artifacts distortions due to field inhomogeneities related to the EPI sequence. Diffusion tensors, color FA map, FA map and MD map are acquired by data reconstruction. Before the next step visual inspection of the undistorted images is highly recommended. ROIs are manually designed on an individual's color FA map in native space. Tractography is conducted for each subject using a deterministic streamline approach with seed FA threshold of 0.2 . In case of more than one participant, ROIs and tracts of interest are automatically reconstructed for all the participants by using a representative subject as a template or an atlas. This resolves user-related variations due to the manual ROI definition. Finally, DTI related metrics such as FA, MD, AD and RD are extracted for each tract

DW: Diffusion Weighted, EPI: Echo-Planar Imaging, FA: Fractional Anisotropy, MD: Mean Diffusivity, ROI: Region of Interest, L: Left, IFOF: Inferior Fronto-occipital Fasciculus, DTI: Diffusion Tensor Imaging, RD: Radial Diffusivity and AD: Axial Diffusivity 


\section{Results}

\section{Demographics and clinical parameters}

Patient subjects and control subjects did not differ significantly in terms of gender $\left[X^{2}(1, \mathrm{~N}=34)=0.424, p=0.515\right]$ and age $(U=138.5, p$ $=0.849, r=0.03)$ (Table 1$)$.

\section{Whole-brain VBM results}

Table 3 presents anatomical regions with reduced GMV in LTLENL compared to HC. Particularly, LTLE-NL patients showed reduced GMV in regions of bilateral mesial temporal, frontal, occipital, and parietal lobes as well as in the cerebellum. None cluster survived in the opposite contrast, LTLE-NL patients GMV > HC GMV. Anatomical regions were identified using WFU-PickAtlas [31,32]. Figure 2 depicts GMV differences in the aforementioned anatomical regions. Results are visualized using the xjView toolbox (http://www.alivelearn.net/xjview).

\section{DTI and tractography results}

MANOVA tests revealed statistically significant differences between the groups on the combined dependent variables for FA $[F(9,24)=$ 3.091, $p=0.013$, Wilks' $\Lambda=0.463$ and $\left.\eta^{2}=0.537\right]$ and $\operatorname{AD}[F(9,24)=$ 2.347, $p=0.046$, Wilks' $\Lambda=0.532$ and $\left.\eta^{2}=0.468\right]$ values, concerning WM fiber tracts ipsilateral to the seizure focus and for FA $[F(2,31)=$ 4.137, $p=0.026$, Wilks' $\Lambda=0.789$ and $\left.\eta^{2}=0.211\right]$ with regard to the pair of Fmajor and Fminor tracts. Particularly, ANOVA tests revealed statistically significant reduced FA values for ipsilateral arcuate $(p=$ $0.045)$, CCGP $(p=0.001), \operatorname{CHP}(p=0.003)$, fornix $(p=0.022)$, IFOF $(p$
$=0.036), \operatorname{ILF}(p=0.010)$ and UNCF $(p=0.032)$ tracts as well as Fmajor $(p=0.007)$ tract in LTLE-NL patients. In addition, LTLE-NL patients showed reduced AD in ipsilateral arcuate $(p=0.028)$ and CHP $(p=$ $0.006)$ tracts compared to HC. No statistically significant differences emerged for the remaining DTI metrics, MD and $\mathrm{RD}$, both ipsilaterally and contralaterally to the seizure focus (Table 4). These bundles are depicted in Figure 3 for both a representative patient and HC subject.

\section{Discussion}

In this study, we investigated the pattern of LTLE-NL patients' structural network alterations using a well-established volumetric analysis and a DTI technique. The homogeneous recruited patient group strengthens our study since there is evidence in the literature that the pathological structural pattern is differentiated when laterality is considered in either TLE-NL or in TLE-HS patients $[1,11,12]$. To the best of our knowledge, there is only one study that has investigated the GM structural integrity of LTLE-NL patients and two studies which have investigated the WM structural integrity, respectively. None of the studies have explored both GM and WM brain structures in the same cohort.

\section{Gray matter integrity}

Regarding the GM, LTLE-NL patients presented widespread GM atrophy in temporal, but in the vast majority also extra-temporal regions. Specifically, LTLE-NL patients showed reduced GMV in bilateral mesial temporal, frontal, occipital, and parietal lobes as well as in the cerebellum. The contribution of extra-temporal regions in TLE-NL

Table 3. Brain anatomical regions with significant GMV differences between LTLE-NL patients and HC groups (LTLE-NL patients GMV < HC GMV)

\begin{tabular}{|c|c|c|c|c|c|}
\hline \multirow{2}{*}{ Brain Lobe } & \multirow{2}{*}{ Anatomical Label } & \multirow{2}{*}{$\begin{array}{l}\text { Hemisphere } \\
(\mathbf{R} / \mathbf{L})\end{array}$} & \multirow{2}{*}{$\begin{array}{l}\text { Coordinates } \\
\text { MNI }(X, Y, Z)\end{array}$} & \multicolumn{2}{|c|}{ Statistics } \\
\hline & & & & Voxels & t-value \\
\hline \multirow[t]{4}{*}{ Frontal lobe } & Precentral Gyrus & $\mathrm{R}$ & $23-2666$ & 36 & 4.43 \\
\hline & Supplementary Motor Area & $\mathrm{L}$ & -10252 & 39 & 3.76 \\
\hline & Supplementary Motor Area & $\mathrm{R}$ & 62057 & 51 & 4.52 \\
\hline & Frontal Sup. Medial Gyrus & $\mathrm{L}$ & -22142 & 23 & 4.78 \\
\hline \multirow[t]{11}{*}{ Occipital lobe } & Middle Occipital Gyrus & $\mathrm{L}$ & $-41-83-14$ & 88 & 4.94 \\
\hline & Middle Occipital Gyrus & $\mathrm{R}$ & $44-8011$ & 548 & 7.83 \\
\hline & Inferior Occipital Gyrus & $\mathrm{L}$ & $-27-98-12$ & 24 & 4.30 \\
\hline & Inferior Occipital Gyrus & $\mathrm{R}$ & $50-77-3$ & 64 & 4.96 \\
\hline & Calcarine Cortex & $\mathrm{L}$ & $-11-98-6$ & 401 & 5.15 \\
\hline & Calcarine Cortex & $\mathrm{R}$ & $5-818$ & 38 & 4.25 \\
\hline & Cuneus & $\mathrm{L}$ & $-3-8326$ & 117 & 4.49 \\
\hline & Cuneus & $\mathrm{R}$ & $11-8338$ & 181 & 4.93 \\
\hline & Lingual Gyrus & $\mathrm{L}$ & $-20-86-17$ & 62 & 6.45 \\
\hline & Lingual Gyrus & $\mathrm{R}$ & $18-635$ & 19 & 4.52 \\
\hline & Superior Occipital Gyrus & $\mathrm{L}$ & $-9-1026$ & 17 & 3.96 \\
\hline \multirow[t]{4}{*}{ Temporal lobe } & Superior Temporal Gyrus & $\mathrm{L}$ & $-62-63$ & 31 & 4.47 \\
\hline & Middle Temporal Gyrus & $\mathrm{R}$ & $47-7511$ & 151 & 6.99 \\
\hline & Inferior Temporal Gyrus & $\mathrm{R}$ & $48-74-6$ & 29 & 5.05 \\
\hline & Fusiform Gyrus & $\mathrm{R}$ & $27-51-15$ & 34 & 3.77 \\
\hline \multirow[t]{3}{*}{ Parietal lobe } & Rolandic Operculum & $\mathrm{L}$ & $-45-2415$ & 22 & 3.73 \\
\hline & Inferior Parietal Gyrus & $\mathrm{R}$ & $32-5042$ & 22 & 3.93 \\
\hline & Supramarginal Gyrus & $\mathrm{R}$ & $59-4435$ & 150 & 5.33 \\
\hline \multirow[t]{7}{*}{ Cerebellum } & Cerebellum Crus 1 & $\mathrm{~L}$ & $-18-86-18$ & 18 & 5.41 \\
\hline & Cerebellum Crus 1 & $\mathrm{R}$ & $30-78-36$ & 72 & 3.96 \\
\hline & Cerebellum Crus 2 & $\mathrm{R}$ & $32-78-39$ & 203 & 4.65 \\
\hline & Cerebellum 6 & $\mathrm{~L}$ & $-32-48-32$ & 16 & 3.64 \\
\hline & Cerebellum 6 & $\mathrm{R}$ & $20-77-21$ & 26 & 4.00 \\
\hline & Cerebellum $7 \mathrm{~b}$ & $\mathrm{~L}$ & $-30-72-48$ & 30 & 4.07 \\
\hline & Cerebellum $7 \mathrm{~b}$ & $\mathrm{R}$ & $38-69-51$ & 35 & 3.80 \\
\hline
\end{tabular}

GMV: Gray Matter Volume, TLE: Temporal Lobe Epilepsy, NL: Non-Lesional, HC: Healthy Controls, R: Right, L: Left, MNI: Montreal Neurological Institute 


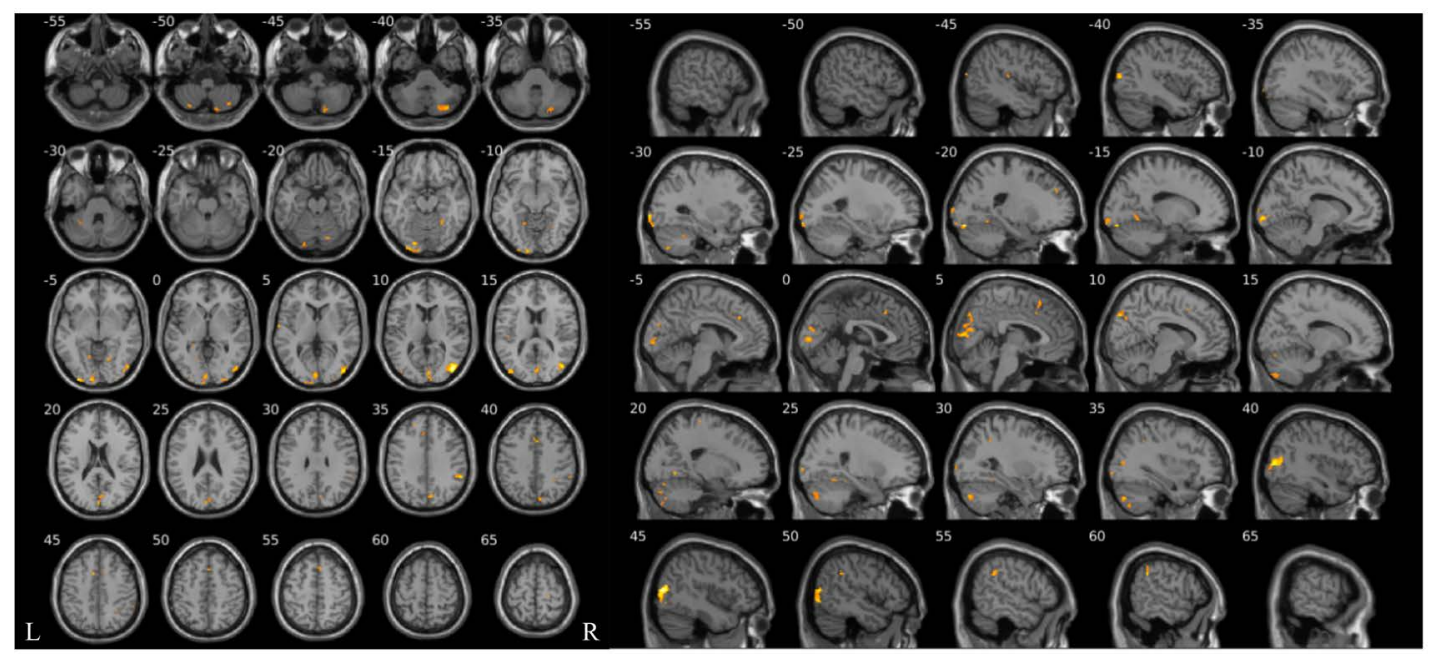

Figure 2. Brain anatomical regions with significant GMV focal atrophy in LTLE-NL patients compared to HC, (LTLE-NL GMV < HC GMV, none cluster survived in the opposite contrast, LTLE-NL patients GMV > HC GMV), in axial (posterior to inferior slice depiction) and sagittal (left to right slice depiction) plane. For illustrative purposes, the findings, depicted by colors, were superimposed on Colin 27 hires T1 template, (MNI space)

GMV: Gray Matter Volume, L: Left, TLE: Temporal Lobe Epilepsy, NL: Non-Lesional and HC: Healthy Controls

Table 4. WM fiber tract differences between LTLE-NL patients and HC groups

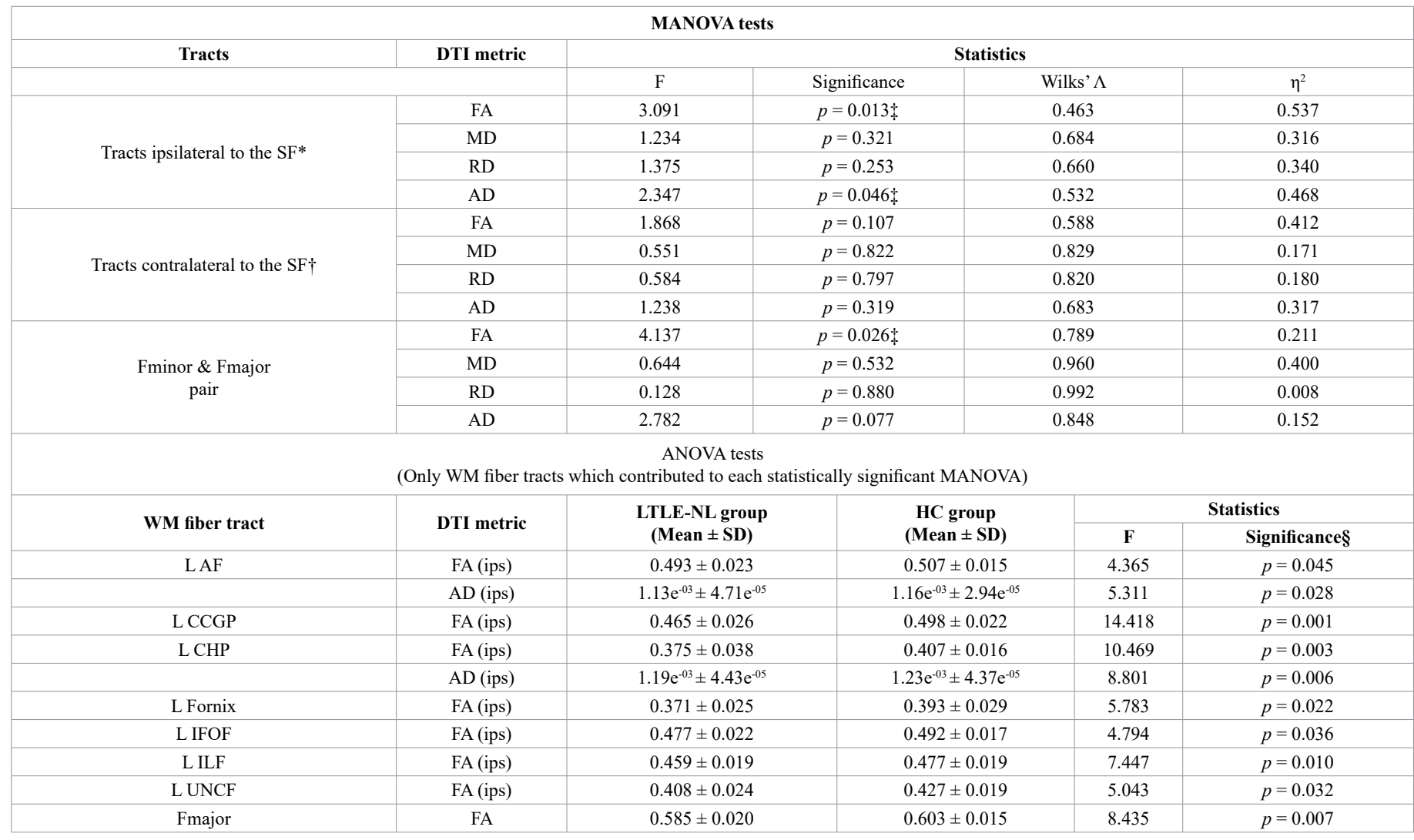

WM: White Matter, TLE: Temporal Lobe Epilepsy, NL: Non-Lesional, HC: Healthy Controls, MANOVA: Multivariate Analysis of Variance, DTI: Diffusion Tensor Imaging, SF: Seizure Focus, FA: Fractional Anisotropy, MD: Mean Diffusivity, RD: Radial Diffusivity, AD: Axial Diffusivity, $\eta^{2}$ : Partial Eta Squared, ANOVA: Analysis of Variance, SD: Standard Deviation, ips: Ipsilateral, L: Left, R: Right, AF: Arcuate Fasciculus, CCGP: Cingulum Cingulate Gyrus Part, CHP: Cingulum Hippocampal Part, IFOF: Inferior Fronto-occipital Fasciculus, ILF: Inferior Longitudinal Fasciculus, UNCF: Uncinate Fasciculus FMajor: Forceps Major and ips: ispilateral.

Data are mean \pm standard deviation. The statistical significance level was set at $p=0.05$.

* Tracts ipsilateral to the SF: L cingulum cingulate gyrus part, L cingulum hippocampal part, L cortico-spinal, L fornix, L anterior thalamic radiation, L arcuate, L inferior longitudinal fasciculus, $\mathrm{L}$ inferior fronto-occipital fasciculus, and L uncinate fasciculus.

† Tracts contralateral to the SF: R cingulum cingulate gyrus part, $\mathrm{R}$ cingulum hippocampal part, $\mathrm{R}$ cortico-spinal, $\mathrm{R}$ fornix, $\mathrm{R}$ anterior thalamic radiation, $\mathrm{R}$ arcuate, $\mathrm{R}$ inferior longitudinal fasciculus, $\mathrm{R}$ inferior fronto-occipital fasciculus, and $\mathrm{R}$ uncinate fasciculus.

$\$$ Statistically significant.

$\S \mathrm{p}$-values are estimated after multiple comparisons (Bonferroni correction). The statistical significance level for the ANOVAs was set at $p=0.05$ because there was one dependent variable. 
Patient Subject

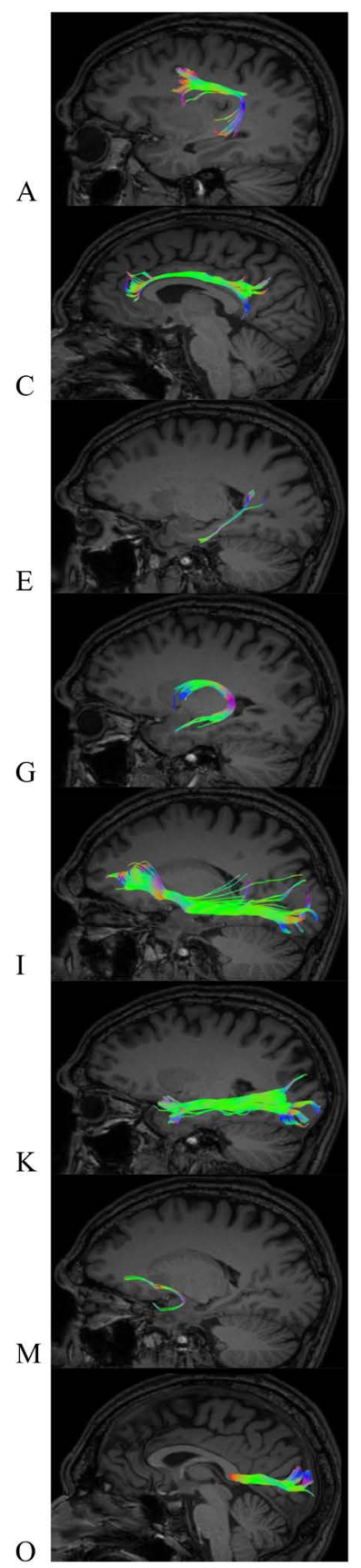

Control Subject

$\mathrm{D}$

$\mathrm{H}$

B

F

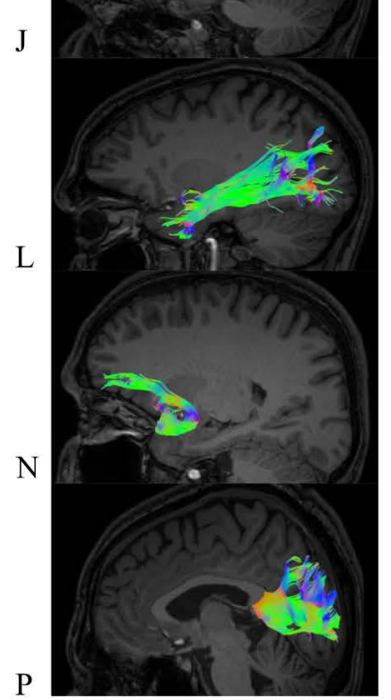

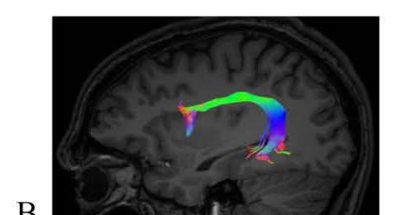

Figure 3. Three-dimensional visualization of WM fiber tracts in a representative LTLENL patient and a representative control subject. Loss of WM fibers is shown in the eight WM fiber tracts which presented statistically significant differences in DTI metrics between LTLE-NL patients and HC and derived through DTI tractography. (A), (B): L arcuate, LAF; (C), (D): L cingulum cingulate gyrus part, L CCGP; (E), (F): L cingulum hippocampal part, L CHP; (G), (H): L fornix; (I), (J): L inferior fronto-occipital fasciculus, L IFOF; (K), (L): L inferior longitudinal fasciculus, L ILF; (M), (N): L uncinate fasciculus, L UNCF and (O) , (P): forceps major, Fmajor

WM: White Matter, L: Left, TLE: Temporal Lobe Epilepsy, NL: Non-Lesional, HC: Healthy Controls

patients' pathophysiology has been indicated in a restricted number of studies $[5,11,33,34]$. Coan, et al. and Mueller, et al. assumed both LTLENL and RTLE-NL patients as a single patient group identifying extended cortical thinning and GM athrophy in both ipsilateral and contralateral temporal and extra-temporal brain areas $[34,35]$. Treating LTLE-NL and RTLE-NL patients as a single group was a study limitation, as referred by one of the above study teams. Nevertheless, findings were in line with the published results of Riederer, et al. who they also identified more extensive and widespread GM changes in LTLE-NL compared to RTLE-NL patients [5]. GM changes in the ipsilateral superior temporal gyrus and in somatosensory and occipital cortices seem to be common findings between our and the aforementioned studies. In addition, we have also found GM abnormalities in the cerebellum that is in concordance with the unique study, in which, LTLE-NL patients were assumed as a separate patient group [5]. Despite the fact that the thalamus is a crucial relay structure and consists common finding in previous GM studies, the present study failed to detect GM atrophy in the thalamus $[5,10,33,34]$. This minor point can be possibly attributed to the administered medication, as well as to the significantly increased standard deviation of the year of onset and disease duration of our patient group. Furthermore, there are references which reveal no GM abnormalities in TLE-NL patients compared to HC. This was probably attributed to the inhomogeneous patient group and to the conducted post-processing analysis methods [9,36]. However, whilst Coan, et al. showed GM differences between TLE-NL and HC groups, the comparison of TLE-NL patients with infrequent seizures with HC didn't identify any GM changes [34]. Thus, the frequency of seizures might be a potential factor of no GM change detection. Synthesizing both our and previous studies' results, we hypothesized that the affected brain structures comprise an intrinsically more widespread epileptogenic network involving extra-temporal brain. Our hypothesis is verified by DTI, functional MRI (fMRI) and dissection studies reporting structural connections between the above mentioned extra-temporal damaged areas and the temporal lobe [37-39].

\section{White matter integrity}

Chronic seizure activity has been implicated in the deterioration of WM fiber tracts which connect the zone of seizure onset with extratemporal focal brain regions [40,41]. Our DTI study confirmed the existence of microstructural WM deteriorations in LTLE-NL patients compared to HC by revealing significant FA reductions in Fmajor and in temporal connective tracts (arcuate, CCGP, CHP, fornix, IFOF, ILF and UNCF) only ipsilateral to the seizure focus which was detected by EEG findings despite the absence of hippocampal atrophy related to TLE-NL. In addition, patients showed reduced AD values in $\mathrm{L}$ arcuate and L CHP tracts. No statistically significant differences emerged for the remaining $\mathrm{MD}$ and $\mathrm{RD}$ DTI metrics, possibly indicating only a restriction of the directionality of the diffusional motion and constituting FA as a more sensitive biomarker. Clinically, the existence of WM alterations only ipsilateral to the EEG abnormalities is extremely important as lateralization is crucial for the presurgical evaluation of patients with TLE.

To the best of our knowledge, there is only one DTI study investigating both LTLE-NL and RTLE-NL patients as distinct groups [13]. Shon, et al. recruited ten LTLE-NL patients and by using a voxelbased technique, different to our DTI analysis approach, verified the ipsilateral lateralization of WM changes (posterior limbic area and parahippocampal region, including parahippocampal cingulum and posterior cingulate cortex). No differences observed in RTLE-NL patients. Ipsilateral (left) temporal findings, reduced FA in fornix, CCGP and CHP fiber tracts, were also confirmed by Liacu, et al. assuming their recruited sample as LTLE-NL group, despite the fact that the seizure onset of one of the nine TLE-NL patients was located in the temporal lobe of the right hemisphere [42]. Hence, we hypothesized 
that the hemisphere in which the seizure onset zone is located was more vulnerable. A covey of studies reinforces this suggestion reporting that LTLE patients present lower learning and memory functions and more widespread WM and GM changes compared to RTLE mainly when the left hemisphere is the dominant language hemisphere $[5,11,14,16,17]$. The above imply that the seizure propagation might be more widespread in this hemisphere probably due to the speculation that left hemisphere is subject to more prolonged maturation than right, resulting to better connectivity and unfortunately to greater vulnerability to early brain malfunctions $[12,18,43,44]$.

However, there are DTI studies which have investigated the TLENL under an alternative approach, assuming both LTLE-NL and RTLENL participants as a single group $[10,41,45,46]$, which was mentioned as a limitation by Liu, et al. This might be considered as the main factor for discrepancies in the literature findings. More precisely, Liu, et al. detected reduced FA only in parahippocampal cingulum and tapetum as well as increased $\mathrm{AD}$ in tapetum [46]. WM changes in tapetum tract were supported by Concha, et al. additionally to the reduced FA in external capsule and Fminor tract. However, they also detected statistically increased values for the rest of DTI metrics (MD, AD and $\mathrm{RD}$ ) in the aforementioned WM fiber tracts [45]. Part of these results are in line with our study results since Fmajor's and tapetum's axons are in close proximity and roughly parallel at the splenium of the corpus callosum. Moreover the external capsule presents long conductive fibers which are part of UNCF and IFOF tracts [47]. Additionally, Vaughan, et al., demonstrated ipsilateral atrophy of the tapetum, UNCF and IFOF as well as bilateral atrophy of CCGP (or dorsal cingulum) and corpus callosum using fiber density and cross-section (FDC) as alternative WM integrity metrics [41,48]. Finally, Scanlon, et al. presented reduced FA in Fminor, body of the corpus callosum and ipsilateral anterior corona radiata using tract-based spatial statistics analysis (TBSS) voxelbased technique [10].

Conversely, our findings are in contrast to a covey of studies which demonstrated no significant WM alterations in TLE-NL patients compared to HC $[9,36,49,50]$. There is a need in the current medical literature for further analysis comprising a larger set of participants, strict patient selection criteria and standardization of novel MRI techniques in order to elucidate on the presence nature and extent of both gray and white matter changes in TLE-NL patients.

\section{Conclusion}

In conclusion, the present study revealed widespread, extratemporal GM atrophies compared to less extensive atrophy in the mesial temporal and limbic structures as well as ipsilateral, widespread, alterations of temporal and extra-temporal WM fiber tracts, in LTLENL patients compared to HC. Therefore, deteriorated afferent and efferent WM fibers of the temporal lobe in conjunction with widespread extra-temporal GM atrophies can shed light on a complex network which might be associated with TLE discharges paving the way for early detection of neuronal loss and accurate localization of the seizure onset zone which are of great clinical significance.

\section{Limitations}

However, the current study was not without limitations. The relatively small sample size and the absence of RTLE-NL patient group will be tackled with recruitment of patients, including an RTLE-NL group in the near future. Moreover, possible effects of antiepileptic medication and seizure frequency and severity were not included in the study analysis due to the fact that this information is often not reliably available. However, our study is strengthened by the strict applied inclusion criteria concerning the seizure laterality and the investigation of both GM and WM brain structures in the same studied group. Therefore, additional clinical data correlations could further enhance the benefit of using VBM and DTI tractography as reliable neuroimaging analysis techniques for the detection of structural network alterations in TLE-NL patients.

\section{Authorship and contributor ship}

Author contributions to the study and manuscript preparation include the following. Conception and study design (Panagiotis Tsialios, Efstratios Karavasilis, Irene Karanasiou, Matilda-Anna Papathanasiou), data collection or acquisition (Efstratios Karavasilis, Panagiotis Tsialios), statistical analysis (Panagiotis Tsialios), interpretation of results (Panagiotis Tsialios, Efstratios Karavasilis, Irene Karanasiou, Anastasios Bonakis, Matilda-Anna Papathanasiou, Georgios Velonakis), drafting the manuscript work or revising it critically for important intellectual content (Panagiotis Tsialios, Efstratios Karavasilis, Matilda-Anna Papathanasiou, Irene Karanasiou, Anastasios Bonakis), approval of the final version to be published and agreement to be accountable for the integrity and accuracy of all aspects of the work (all authors).

\section{Acknowledgments}

We wish to thank all patients and healthy participants for their willingness to participate in the present study.

\section{Funding sources}

There is no funding for this study.

\section{Compliance with ethical standards}

All procedures performed in this study, involving human participants, were in accordance with the ethical standards of the institutional and/or national research committee and with the 1964 Helsinki Declaration and its later amendments or comparable ethical standards.

\section{Conflict of interest}

The authors report no conflicts of interest.

\section{Informed consent}

Informed consent was obtained from all individual participants included in the study.

\section{References}

1. Whelan CD (2018) Structural brain abnormalities in the common epilepsies assessed in a worldwide ENIGMA study. Brain 141: 391-408.

2. Aaron AC (2005) Normal magnetic resonance imaging and medial temporal lobe epilepsy: the clinical syndrome of paradoxical temporal lobe epilepsy. Journal of Neurosurgery 102: 902-909. [Crossref]

3. Cascino GD (1991) Magnetic resonance imaging-based volume studies in temporal lobe epilepsy: Pathological correlations. Annals of Neurology 30: 31-36. [Crossref]

4. Kim SE (2006) The clinical and electrophysiological characteristics of temporal lobe epilepsy with normal MRI. Journal of Clinical Neurology 2: 42-50. [Crossref]

5. Riederer F (2008) Network atrophy in temporal lobe epilepsy. Neurology 71: 419.

6. Wang (2013) The pathology of magnetic-resonance-imaging-negative epilepsy Modern Pathology 26: 1051.

7. Kanemoto (1996) Characteristics of temporal lobe epilepsy with mesial temporal sclerosis, with special reference to psychotic episodes. Neurology 47: 1199. 
8. Labate A (2016) Long-term outcome of mild mesial temporal lobe epilepsy. Neurology 86: 1904 .

9. Mueller (2006) Voxel-based optimized morphometry (VBM) of Gray and white matter in Temporal Lobe Epilepsy (TLE) with and without Mesial Temporal Sclerosis. Epilepsia 47: 900-907.

10. Scanlon (2013) Grey and white matter abnormalities in temporal lobe epilepsy with and without mesial temporal sclerosis. Journal of Neurology 260: 2320-2329.

11. Ahmadi ME (2009) Side matters: diffusion tensor imaging tractography in left and right temporal lobe epilepsy. AJNR. American Journal of Neuroradiology 30: 1740-1747.

12. Kemmotsu N (2011) MRI analysis in temporal lobe epilepsy: cortical thinning and white matter disruptions are related to side of seizure onset. Epilepsia 52: 2257-2266.

13. Shon YM (2010) Group-specific regional white matter abnormality revealed in diffusion tensor imaging of medial temporal lobe epilepsy without hippocampal sclerosis. Epilepsia 51: 529-535.

14. Bell BD (1998) Anterior Temporal Lobectomy, Hippocampal Sclerosis, and Memory: Recent Neuropsychological Findings. Neuropsychology Review 8: 25-41.

15. Helmstaedter C (2009) Chronic temporal lobe epilepsy: a neurodevelopmental or progressively dementing disease? Brain 132: 2822-2830.

16. Bonilha L (2007) Asymmetrical extra-hippocampal grey matter loss related to hippocampal atrophy in patients with medial temporal lobe epilepsy. Journal of Neurology, Neurosurgery, and Psychiatry 78: 286-294.

17. Coan AC (2009) Seizure frequency and lateralization affect progression of atrophy in temporal lobe epilepsy. Neurology 73: 834.

18. Corballis M (2010) On the biological basis of human laterality: I. Evidence for a maturational left-right gradient. Behavioral and Brain Sciences 1: 261-269.

19. Njiokiktjien C (2006) Differences in vulnerability between the hemispheres in early childhood and adulthood. Fiziol Cheloveka 32: 45-50.

20. Pujol J (2006) Myelination of language-related areas in the developing brain. Neurology 66: 339-343.

21. Sone D (2018) Abnormal neurite density and orientation dispersion in unilateral temporal lobe epilepsy detected by advanced diffusion imaging. NeuroImage Clinical 20: $772-782$.

22. Leemans A (2009) ExploreDTI: a graphical toolbox for processing, analyzing, and visualizing diffusion MR data. in 17th Annual Meeting of Intl Soc Mag Reson Med.

23. Perrone D (2015) The effect of Gibbs ringing artifacts on measures derived from diffusion MRI. NeuroImage 120: 441-455.

24. Tax CMW (2015) REKINDLE: Robust extraction of kurtosis INDices with linear estimation. Magnetic Resonance in Medicine 73: 794-808.

25. Jones DK (2010) Twenty-five pitfalls in the analysis of diffusion MRI data. NMR in Biomedicine 23: 803-820.

26. Jones DK, Leemans A (2011) Diffusion Tensor Imaging, in Magnetic Resonance Neuroimaging: Methods and Protocols, M. Modo and J.W.M. Bulte, Editors. Humana Press: Totowa, NJ. p. 127-144

27. Lawes INC (2008) Atlas-based segmentation of white matter tracts of the human brain using diffusion tensor tractography and comparison with classical dissection. NeuroImage 39: 62-79. [Crossref]

28. Wakana S (2007) Reproducibility of quantitative tractography methods applied to cerebral white matter. NeuroImage 36: 630-644.
29. Hayasaka S (2004) Combining voxel intensity and cluster extent with permutation test framework. NeuroImage 23: 54-63.

30. Worsley KJ (1999) Detecting changes in nonisotropic images. Human Brain Mapping 8: $98-101$.

31. Maldjian JA, Laurienti PJ, Burdette JH (2004) Precentral gyrus discrepancy in electronic versions of the Talairach atlas. NeuroImage 21: 450-455.

32. Maldjian JA (2003) An automated method for neuroanatomic and cytoarchitectonic atlas-based interrogation of fMRI data sets. NeuroImage 19: 1233-1239.

33. Alvim MKM (2016) Progression of gray matter atrophy in seizure-free patients with temporal lobe epilepsy. Epilepsia 57: 621-629.

34. Coan AC (2014) Frequent seizures are associated with a network of gray matter atrophy in temporal lobe epilepsy with or without hippocampal sclerosis. PloS one 9: e85843-e85843.

35. Mueller SG (2009) Widespread neocortical abnormalities in temporal lobe epilepsy with and without mesial sclerosis. NeuroImage 46: 353-359.

36. Beheshti I (2018) Gray Matter and white matter abnormalities in temporal lobe epilepsy patients with and without hippocampal sclerosis. Frontiers in Neurology 9.

37. Catani M (2003) Occipito-temporal connections in the human brain. Brain. 126: 2093 2107.

38. Menjot de Champfleur (2013) Middle longitudinal fasciculus delineation within language pathways: A diffusion tensor imaging study in human. European Journal of Radiology 82: 151-157.

39. Sokolov AA (2012) Structural loop between the cerebellum and the superior temporal sulcus: Evidence from diffusion tensor imaging. Cerebral Cortex 24: 626-632. [Crossref]

40. Concha L (2014) A macroscopic view of microstructure: Using diffusion-weighted images to infer damage, repair, and plasticity of white matter. Neuroscience 276: 14-28.

41. Vaughan DN (2017) Tract-specific atrophy in focal epilepsy: Disease, genetics, or seizures? Annals of Neurology 81: 240-250.

42. Liacu D (2012) Diffusion tensor imaging tractography parameters of limbic system bundles in temporal lobe epilepsy patients. Journal of Magnetic Resonance Imaging 36: $561-568$.

43. Focke NK (2008) Voxel-based diffusion tensor imaging in patients with mesia temporal lobe epilepsy and hippocampal sclerosis. NeuroImage 40: 728-737. [Crossref]

44. Powell HWR (2007) Reorganization of verbal and nonverbal memory in temporal lobe epilepsy due to unilateral hippocampal sclerosis. Epilepsia 48: 1512-1525.

45. Concha L (2009) White-matter diffusion abnormalities in temporal-lobe epilepsy with and without mesial temporal sclerosis. Journal of Neurology, Neurosurgery \& Psychiatry 80: 312.

46. Liu M (2012) Mesial temporal sclerosis is linked with more widespread white matter changes in temporal lobe epilepsy. NeuroImage Clinical 1: 99-105.

47. Wakana S (2004) Fiber Tract-based atlas of human white matter anatomy. Radiology 230: 77-87.

48. Raffelt, DA (2017) Investigating white matter fibre density and morphology using fixelbased analysis. NeuroImage 144: 58-73. [Crossref]

49. Campos BM (2015) White matter abnormalities associate with type and localization of focal epileptogenic lesions. Epilepsia 56: 125-132.

50. Gong G (2008) Thalamic diffusion and volumetry in temporal lobe epilepsy with and without mesial temporal sclerosis. Epilepsy Research 80: 184-193. [Crossref]

Copyright: (C2020 Tsialios P. This is an open-access article distributed under the terms of the Creative Commons Attribution License, which permits unrestricted use, distribution, and reproduction in any medium, provided the original author and source are credited. 